\title{
QUEEN'S
UNIVERSITY
BELFAST
}

\section{Experimental evaluation of the response of micro-channel plate detector to ions with 10 s of MeV energies}

Jeong, T. W., Singh, P. K., Scullion, C., Ahmed, H., Kakolee, K. F., Hadjisolomou, P., Alejo, A., Kar, S., Borghesi, M., \& Ter-Avetisyan, S. (2016). Experimental evaluation of the response of micro-channel plate detector to ions with $10 \mathrm{~s}$ of MeV energies. Review of Scientific Instruments, 87(8), [083301]. https://doi.org/10.1063/1.4959187

Published in:

Review of Scientific Instruments

Document Version:

Peer reviewed version

Queen's University Belfast - Research Portal:

Link to publication record in Queen's University Belfast Research Portal

Publisher rights

(C) 2016 AIP Publishing LLC. This article may be downloaded for personal use only. Any other use requires prior permission of the author and AIP Publishing.

The following article appeared in Jeong, TW, Singh, PK, Scullion, C, Ahmed, H, Kakolee, KF, Hadjisolomou, P, Alejo, A, Kar, S, Borghesi, M \& Ter-Avetisyan, S 2016, 'Experimental evaluation of the response of micro-channel plate detector to ions with 10 s of MeV energies' Review of Scientific Instruments, vol 87, no. 8, 083301, and may be found at http://scitation.aip.org/content/aip/journal/rsi/87/8/10.1063/1.4959187

\section{General rights}

Copyright for the publications made accessible via the Queen's University Belfast Research Portal is retained by the author(s) and / or other copyright owners and it is a condition of accessing these publications that users recognise and abide by the legal requirements associated with these rights.

\section{Take down policy}

The Research Portal is Queen's institutional repository that provides access to Queen's research output. Every effort has been made to ensure that content in the Research Portal does not infringe any person's rights, or applicable UK laws. If you discover content in the

Research Portal that you believe breaches copyright or violates any law, please contact openaccess@qub.ac.uk. 


\title{
Experimental evaluation of the response of Micro Channel Plate detectors to ions with 10s of MeV energies
}

\author{
Tae Won Jeong ${ }^{1,2}$, P. K. Singh ${ }^{1}$, C. Scullion ${ }^{3}$, H. Ahmed ${ }^{3}$, K. F. Kakolee ${ }^{1}$, \\ P. Hadjisolomou ${ }^{3}$, A. Aaron ${ }^{3}$, S. Kar ${ }^{3}$, M. Borghesi ${ }^{3}$, and S. Ter-Avetisyan ${ }^{1,2}$ \\ ${ }^{1}$ Center for Relativistic Laser Science, Institute of Basic Science (IBS), Gwangju 61005, Korea \\ ${ }^{2}$ Department of Physics and Photon Science, Gwangju Institute of Science and Technology (GIST), \\ Gwangju 61005, Korea \\ ${ }^{3}$ School of Mathematics and Physics, The Queen's University of Belfast, Belfast, BT7 1NN, UK
}

The absolute calibration of a microchannel plate (MCP) assembly using a Thomson spectrometer for laserdriven ion beams is described. In order to obtain the response of the whole detection system to the particles' impact, a slotted solid state nuclear track detector such as CR-39 was installed in front of MCP to record the ions simultaneously on the CR-39 and the MCP. The response of the MCP (counts/particles) was measured for 5 $-58 \mathrm{MeV}$ carbon ions and for protons in the energy range $2-17.3 \mathrm{MeV}$.

The response of the MCP detector is non-trivial when the stopping range of particles becomes larger than the thickness of the detector. Protons with energies $E \gtrsim 10 \mathrm{MeV}$ are energetic enough that they can pass through the MCP detector. Quantitative analysis of the pits formed in CR-39 and the signal generated in the MCP allowed to determine the MCP response to particles in this energy range. By reproducing the experimental observation with a theoretical model allows to prediet the response of MCP at even higher proton energies. This suggests that in this regime the MCP response is a slowly decreasing function of energy, consistently with the decrease of the deposited energy. This calibration data will enable particle spectra to be obtained in absolute terms over a broad energy range.

\section{INTRODUCTION}

High power lasers with intensities exceeding $10^{21} \mathrm{~W} / \mathrm{cm}^{2}$ are an efficient tool for accelerating multispecies charged particles to relativistic energies [1]. Laser-driven ion beams are polychromatic and have wide energy spread. To characterize these particles the Thomson spectrometer is widely used ([2] and reference within), which provides the distribution of accelerated ions as a function of their energy and mass-to-charge ratio, simultaneously. Numerous detectors have been used in the past decades to record high-energy charged particles. These include solid state nuclear track detectors such as CR-39, [3] nuclear emulsions, radiochromic films (RCFs), [4] image/phosphor plates, [5] scintillators, Cherenkov detectors, Faraday cups. The Thomson spectrometer becomes a particularly valuable diagnostic if a microchannel plate (MCP) detector coupled to the phosphor screen is used. The MCP detector enables real time operation of the Thomson spectrometer with single particle sensitivity. However, for a quantitative analysis of the ion beams the absolute calibration of detection system is needed.

Recently the Thomson spectrometer - MCP assembly was calibrated for neutral atoms and positive and negative ions with energies in the $\mathrm{MeV}$ range [6,7]. This paper describes the calibration of the generated actual signal strength of the recorded event of charged particles impact, called response of MCP detector, at significantly higher energies than studied previously. Particularly, the calibration becomes non trivial when the 
stopping range of particles becomes larger than the thickness of the detector, or, in other words, the detector cannot stop those particles. In fact, the MCP plates made of lead-silicate glass (main material) with typical thicknesses of up to $0.6 \mathrm{~mm}$ the protons with energies above $10 \mathrm{MeV}$ cannot be stopped [8] whereas nowadays experimentally observed ions/protons reach energies of tens of $\mathrm{MeV}$ per nucleon. Careful consideration is required in evaluating the MCP signal in this regime, since the gain and the secondary electron yield from the MCP plate can be affected, and constraints are posed on the calibration procedure. A theoretical model, in good agreement with the experimental calibration data, allowed extending the calibration to several tens of $\mathrm{MeV}$ ions. The calibration of the detection system is essential for a correct quantitative analysis and interpretation of the experimental data.

\section{EXPERIMENTAL SETUP}

The charged particles were generated by irradiating foil targets with the Ti-Sapphire laser beam at the Center for Relativistic Laser Science (CoReLS), GIST, Gwangju [9]. In this experiments, 30 fs laser pulses of energy $14 \mathrm{~J}$ were focused by a $\mathrm{f} / 3$ off axis parabolic mirror at a peak intensity of $\sim 2 \times 10^{20} \mathrm{~W} / \mathrm{cm}^{2}$ onto $6 \mu \mathrm{m} \mathrm{Al}$ targets at an incidence angle of $30^{\circ}$ to the target normal. In these experiments, the measurements of the ion spectrum were carried out with a Thomson spectrometer. Typically, a magnetic field of $(0.9 \pm 0.1) \mathrm{T}$ and electric field up to $22 \mathrm{kV} / \mathrm{cm}$ were applied. The ions were detected by an MCP detector [10], with a diameter of $75 \mathrm{~mm}$ coupled to a phosphor screen $(\mathrm{P} 43)$ that was imaged with a 2 -inch objective having $50 \mathrm{~mm}$ focal length and F0.95 aperture onto the chip of a 16 bit CCD camera (PIXIS 1024, Princeton Instruments). The effective f-number of the imaging system was F6. Generated digital signal in CCD chip was about few 1000 counts implying a linear response of CCD. The entrance pinhole of the spectrometer was $100 \mu \mathrm{m}$, which ensures that the ion species are clearly resolved on the detector and the ion spectra can be recorded with high energy resolution $(\sim 0.7 \%$ at 20 $\mathrm{MeV}$ per nucleon). The solid angle for ion collection was $4.7 \times 10^{-9} \mathrm{sr}$.

In order to calibrate the response of the detection system to the impact of energetic charged particles, a $1 \mathrm{~mm}$ thick slotted CR-39 (solid state nuclear track detectors) plate having identical width and spacing of 4 mm were installed in front of the MCP detector (see Fig. 1) [6] in a single or double layer configurations. Track formation in CR-39 takes place by the passage of energetic particle via an electronic collision cascade process, which spreads outward from the particle trajectory, breaks the molecular structures and results in a damaged zone in the plate [11]. After exposure to the accelerated ions, the CR-39 was etched in a $6 \mathrm{~N} \mathrm{NaOH}$ solution at a constant temperature of $68.5^{\circ} \mathrm{C}$. The etching process reveals damaged zones, generally referred as pits, due to the impact of particles. Thus, recorded parabolic traces of ions [6] appear on the stripes of CR-39 and on the MCP between the stripes, shown in Fig. 2. The entire etching process was carried out in small time intervals to avoid any overlapping of the pits. After each etching step, the pits were counted manually under an optical microscope.

\section{CALIBRATION METHOD}

The parabolic ion traces, simultaneously recorded on the CR-39 and on the MCP, are shown in Fig. 2(a) and 2(b), respectively. The "zero point" signal indicated by the arrow in Fig. 2(b) results from emitted radiation from the source which is unaffected by the electric and magnetic fields of the spectrometer (neutral particles and $\mathrm{X}$-rays). The inset in the Fig. 2(a) shows as an example the $20 \times$ magnified image of the $\mathrm{C}^{6+}$ pits at the energies around 9.4 MeV recorded by an optical microscope. The Thomson spectrometer arrangement employed, having 
high charged species discrimination (separation by $Q / M, Q-$ charge, $M$ - mass) and high energy resolution, required a small entrance pinhole $(100 \mu \mathrm{m})$, which restricted the number of recorded particles. Therefore, in order to improve the statistics, namely the pits on the CR-39, three consecutive laser shots were accumulated on CR-39 while the MCP images were recorded on a single shot basis and were summed up afterwards after subtracting the background. Additionally, the number of particles counted on CR-39 and registered counts on CCD image as a digitalised response of MCP signal have been binned over $1 \mathrm{~mm}$-wide steps. The latter gives the energy resolution about $3 \%$ at $2.5 \mathrm{MeV}$ and about $8 \%$ at $17 \mathrm{MeV}$ energies. Exclusion of data points at the edges of CR-39 stripes led to three data points in each of the $4 \mathrm{~mm}$-wide CR-39 stripes as well as in the adjacent trace segment (transmitted through the slots) revealed in the MCP. The signal registered on the CCD camera in the region between the CR-39 stripes were compared to the number of particles that were counted on the CR-39 stripes after etching. Similarly to the procedure reported in [6], the relative calibration was done by assuming that the number of particles on adjacent stripes correlates with the CCD integrated counts between the stripes and belongs to the same parabolic trace. Having three data points on each stripe allows to extrapolate with good accuracy the number of protons between the stripes.

The stopping range of protons with energies $>10 \mathrm{MeV}$ is larger than the $1 \mathrm{~mm}$ thickness of the CR-39 plate we employed [8]. For that reason two configurations of detectors were used, i.e. one and two layers of CR-39 placed in front of the MCP. The second configuration allows to record particles distribution between first and second layers of CR-39 in order to infer particle number detected by the MCP in the back of CR-39 stripe in the single layer CR-39 and MCP configuration with the energies for which a single CR-39 layer is not enough in order to stop those particles.

In the configuration using two layers of slotted CR-39, the MCP shows a dark shadow in correspondence of the CR-39 stripes (Fig. 2(b)) which indicates the total stopping of the particles in the CR-39s. It is worth to mention that in the experiments pit formation due to particles impact is observed along the whole thickness of CR-39 plate (Fig. 2(a)): At low energies $(E<4.8 \mathrm{MeV})$ the protons are stopped at the front surface of the detector, and as the energies increase (next stripe covers the energy interval $6 \mathrm{MeV}<E<7.3 \mathrm{MeV}$ ) the pits appear in the interior of CR-39 (which can be revealed with extensive etching). At about $E \gtrsim 9.5 \mathrm{MeV}$ (next stripe) the pits are formed on the rear surface of the CR-39 plate. With a further energy increase $(E \gtrsim 10 \mathrm{MeV})$ pits begin to appear also on the second CR-39 layer (Fig. 3(a)). The protons in the energy range $10 \mathrm{MeV}<E<$ 12.3 MeV (restricted by energy range on the stripe) have created pits on the rear side of the first CR-39 and on the front side of the second CR-39 plate. Qualitatively, the analysis of the pits is shown in Fig. 3(b) together with the fraction of transmitted protons through the first CR-39 plate as a function of energy.

In the experiments where a single plate of slotted CR-39 was installed in front of the MCP detector the information above is useful to interpret the signal observed on the MCP behind a CR-39 stripe which can partly or fully stop those particles.

The energy calibration of the segmented parabolic traces (Fig. 2) was obtained using a MATLAB routine. The parabolic traces are described analytically based on approximation of small deflections assuming that the both fields in average are uniform over the length of the plates and zero outside, and the ions are non-relativistic [12]. Partially segmented spectra on CR-39 and MCP were individually fitted with the help of polynomial fitting function. Finally the calibration results (counts/particles) were obtained by dividing the MCP functional 
fitting curve to that of CR-39. Main sources of error in the MCP calibration factor were Poisson's distribution of signal counting and the curve fitting procedure.

\section{RESULTS AND DISCUSSION}

\section{- Calibration of protons}

In Fig. 4 a correlation of integrated particles counts in CR-39 stripes and MCP signal between the stripes is shown. These data were obtained in three consecutive shots, which were accumulated on a stack of two CR-39s, while the MCP signal is obtained by summing the MCP raw data for these shots. Protons having energies $E<10 \mathrm{MeV}$, due to their total stopping in $1 \mathrm{~mm}$ thickness of CR-39, were counted from the first layer of CR39, and the protons with $E>10 \mathrm{MeV}$ energies from the second CR-39 plate. Because the etching was carried out in small time intervals the pits have been revealed and counted along the whole thickness of CR-39 and at the rear surface upon their appearance.

As discussed earlier, in $1 \mathrm{~mm}$-thick CR-39 the particles with energies $E>10.4 \mathrm{MeV}$ [8] cannot be stopped. Experiments showed that the protons at these energies are creating pits on the rear surface of the first CR-39 plate, and on the front surface of the second CR-39 plate (Fig. 3(a)). The number of pits on both CR-39 layers in this transparency regime is almost the same (Fig. 3(b)), although the width of the spectral trace on the second layer is slightly broader than on the first layer, which is due to the scattering of the protons while passing through the first CR-39. Appearance of the pits on both CR-39 plates at the same particles energies $(10 \mathrm{MeV}<$ $E<10.3 \mathrm{MeV}$ ) for which $1 \mathrm{~mm}$ thickness of CR-39 tends do not be stopped confirms the probabilistic nature of the stopping range of the particles within the Bragg peak. The equal amount of particles measured on both CR39 plates (back of first plate and front of second) at the energies $10.3 \mathrm{MeV}<E<10.7 \mathrm{MeV}$ suggests that although the first layer of CR-39 cannot stop those particles the deposited energy is enough to damage the plate and create pits. Additionally, particles creating pits in the first CR-39 are slowed down (e.g., $10.6 \mathrm{MeV}$ protons are slowed down to $2.3 \mathrm{MeV}$ ) and will be fully stopped in the second CR-39 plate. Thus, in the transient mode when the energy of the particle increases and its stopping range becomes greater than the thickness of the first detector, each proton creates a pit in both layers of CR-39s.

In the configuration where only one plate of CR-39 was employed the transmitted protons through the CR39 were recorded by the MCP. In the inset of Fig. 5(b) the magnified image of MCP signal in the $10-12.6 \mathrm{MeV}$ energy range is shown, which is located under the corresponding stripe in the CR-39 plate. The signal was produced by the protons which have passed through a $1 \mathrm{~mm}$ thick CR-39 plate and as a result have been slowed down to energies $2-6.6 \mathrm{MeV}$ [8]. Therefore, one cannot assign these data to the calibration of MCP for high energies, however they can be used as confirmation of the calibration at this energy interval, because, as we have seen from double layer CR-39 data, the amount of protons recorded on both CR-39 plates were the same. Therefore, the applied method for MCP calibration is restricted by the stopping range of particles larger than detector thickness.

The proton signal on the MCP in the energy range $18 \mathrm{MeV}<E<20 \mathrm{MeV}$, which is located behind a CR39 stripe, can be clearly seen (see inset in Fig. 5(b)), whereas, no pits were observed on the corresponding CR39 stripe. This implies that the CR-39 becomes completely transparent for protons of energies $E>18 \mathrm{MeV}$. In contrast, the $0.6 \mathrm{~mm}$ thick MCP plate, although it cannot stop even the slowed down protons in CR-39 (e.g., 18 
- $20 \mathrm{MeV}$ protons become $14-16.5 \mathrm{MeV}$, and MCP plate can pass the protons above $8 \mathrm{MeV}$ ), could still generate a sufficient amount of signal, which is consistent with the MCP response in this part of the spectra. It is interesting to note, that there is no observable broadening of the spectral trace in this part of the spectra (Fig. 5) in contrast to the $10-12.6 \mathrm{MeV}$ energy range (Fig. 3) where the width of the spectral trace on the second layer was broadened compare to the first layer, as it was discussed above. Simulations of the propagation of ions in matter [8] shows slight broadening (in a range of $\pm 10 \mu \mathrm{m}$ ) which is below our resolution. Therefore, it is suggestive, that the detection efficiency of MCP for high energy ions can be increased by slowing down of ions in the appropriately chosen medium where the scattering of ions for a given measurement setup can be neglected.

The Fig. 6 shows the calibration factor for protons, for both the single and the three accumulated shots. The calibration factor could be obtained experimentally up to about $17.3 \mathrm{MeV}$. The MCP response for protons is very similar or the same for energies $>18 \mathrm{MeV}$ and 14-16.5 MeV. The latter means that the energy deposition of those protons is very similar which is because the protons are not in the Bragg peak part of their energy deposition and therefore the energy deposited is almost similar. It will be shown later in this paper that on the basis of theoretical considerations with this assumption the calibration curve for the sensitivity of the MCP to particle impact can be extended to much higher energies.

\section{- Calibration of carbon ions}

In the same way, the response of MCP to carbon ions including $\mathrm{C}^{4+}, \mathrm{C}^{5+}$ and $\mathrm{C}^{6+}$ was obtained as shown in Fig. 7. In this case single shot data have been used due to the following reasons; in spite of the number of carbon ions being about 3 times lower than protons at the same energy/nucleon, all the carbon ions are stopped on the surface of CR-39, and the size of the pits were about 5 times bigger than that created by protons which leads easily to saturation of the plates. The $\mathrm{MCP}$ response is similar for all measured carbon ions $\mathrm{C}^{4+}, \mathrm{C}^{5+}$ and $\mathrm{C}^{6+}$ over a broad energy range (Fig. 7), i.e. there is no charge state dependence of the response.

Fig. 8(a) shows the raw image of ion traces obtained by employing a Thomson spectrometer coupled to the MCP detector along the target normal direction in the same experimental setup and interaction geometry at a laser intensity of about $2 \times 10^{20} \mathrm{~W} / \mathrm{cm}^{2}$ focused on $6 \mu \mathrm{m} A l$ target. The proton and Carbon spectra obtained using the calibration factor obtained according to the procedure explained above are shown in Fig.8a.

\section{THEORETICAL MODEL}

The theoretical model considered in [6] was used to simulate the generated signal strength in MCP to the proton and carbon ion impact depending on their energy. Similarly, adopting the general expression for the secondary electron yield from a solid [13], and assuming that for a particular material the probability of electron escape from the surface $(P)$, the energy needed to be deposited to produce an electron for escape $\left(E_{0}\right)$, and the characteristic length of electron diffusion inside the material are constant $(\lambda)$, the response of the MCP on particles impact can be written as

$$
R_{M C P} \propto g \cdot \frac{1}{\cos \theta} \int_{0}^{L}\left(\frac{d E}{d x}\right)_{e} d x
$$

where $(d E / d x)_{e}$ is the electronic stopping power of the projectile ion into the material and $\theta$ is the angle of incidence with respect to the normal to the MCP cannel surface, $g$ is the gain in MCP channel which could be 
approximated as an exponential function on channel length $(L)$ and applied voltage, and it can be calculated as $g=e^{k\left(L-x_{L}\right) / L}$, where $k$ is a constant, $x_{L}$ is the ion penetration depth inside the channel.

The stopping power $(d E / d x)_{e}$ of the particles has been considered at different energies to calculate the gain. For protons, the stopping range increases significantly with the particle energies. Although the stopping range of $E>10 \mathrm{MeV}$ protons is larger than MCP thickness, a detectable signal is generated in the MCP because the amount of energy released in the material is still higher than the energy required for releasing an electron $\left(E_{0}\right)$. Therefore, the MCP response was calculated as

$$
R_{M C P} \propto \frac{1}{\cos \theta} \sum_{i=0}^{L}\left(\frac{d E}{d x}\right)_{i} \Delta x_{i} g_{i}
$$

where the energy is summed over the length of MCP with the corresponding change of the gain in the channel, with the assumption, that the released energy in the material is proportional to the produced electrons for escape. The incidence angle $\theta$ of particles is known from the geometry of the experiments. For instance, for protons $\theta \sim 80.4^{\circ}$ at $2.5 \mathrm{MeV}$ and $87.5^{\circ}$ at $40 \mathrm{MeV}$ and for carbon ions $\theta \sim 80.9^{\circ}$ at $8.4 \mathrm{MeV}$ and $87.1^{\circ}$ at $84 \mathrm{MeV}$. The experimentally obtained calibration results for protons is compared to the theoretical model in Fig. 6. The model reproduces reasonably well the experimental observation along the whole experimental range up to 17.3 $\mathrm{MeV},[13]$ and predicts that at even higher energies the response of MCP will decrease very slowly with energy, in connection with the decrease of the deposited energy.

Regarding the response of MCP to carbon ions, we observe that their stopping range is very short for the energies we could produce experimentally (e.g. for $60 \mathrm{MeV}$ carbon ions the stopping range is $76 \mu \mathrm{m}$ ), therefore, varying the stopping power with energy does not significantly contribute to the response, and consequently to the gain in MCP channel. As a result, the dependence on $1 / \cos \theta$ is the main cause of variation in response for different Carbon energies. As shown in Fig. 7(b) the theoretical model reproduces the experimental results well also for Carbon ions. It is expected that for higher energies, above $200 \mathrm{MeV}$, having significantly longer penetration depth, the MCP will show a similar response as observed for protons.

\section{CONCLUSIONS}

In conclusion, we have discussed the method and results of absolute calibration of the MCP assembly detector using the Thomson spectrometer and slotted CR-39. The absolute calibration data gives a relation between the numbers of particles impact on MCP and counts on the CCD image of the phosphor screen. The MCP response (counts/particles) of protons and carbon ions were presented for the energy range $2-17.3 \mathrm{MeV}$ for protons and 5-58 MeV for carbon ions. The difference in response of the MCP detector to carbon ions and protons results from the different propagation characteristics (within the energy ranges investigated) of the particles inside the MCP. We have analysed the response of the detectors (CR-39 and MCP) to the more energetic particles in the spectrum when detectors become cannot stop those particles. Experiments showed that the detection efficiency of MCP for high energy ions can be increased by slowing down of ions in the appropriately chosen medium where the scattering of ions for a given measurement setup can be neglected.

The experimental results are compared with model presented in Ref. [2]. The results of model calculation and experimental data are in a good agreement with each other, and allow extrapolating the response to higher energies, with the extrapolation showing only a very slow dependence on particle energy. 
The transparency of the CR-39 plate constitutes a limitation of the method for MCP calibration presented in this paper. We discuss here how the energy range of protons can be extended further considering the stopping range of protons in thicker layers or stacks of multiple CR-39s. Once a suitable calibration is performed, this sensitive detection set up makes it possible to measure an absolute ion energy spectrum in a single laser shot.

\section{ACKNOWLEDGMENTS}

The authors would like to thank technical support of Mr. C. J. Im, Mr. C. W. Kim, Mr. S. Y. Kim, and Mr. S. W. Kang and CoReLS laser team for the experiment. This work was supported by the Institute for Basic Science (IBS) under IBS-R012-D1. The authors acknowledge funding from EPSRC, through grants EP/J002550/1, EP/L002221/1, EP/K022415/1, EP/J500094/1. 
This manuscript was accepted by Rev. Sci.Instrum. Click here to see the version of record.

\section{REFERENCES}

[1] A. Macchi, M. Borghesi, and M. Passoni. Rev. Mod. Phys. 85, 751 (2013)

[2] S. Ter-Avetisyan, B. Ramakrishna, D. Doria, G. Sarri, M. Zepf, M. Borghesi, L. Ehrentraut, H. Stiel, S. Steinke, G. Priebe, M. Schnürer, P. V. Nickles, and W. Sandner, Rev. Sci. Instrum. 80, 103302 (2009).

[3] F. H. Seguin, J. A. Frenje, C. K. Li, D. G. Hicks, S. Kurebayashi, J. R. Rygg, B.-E. Schwartz, R. D. Petrasso, S. Roberts, J. M. Soures, D. D.Meyerhofer, T. C. Sangster, J. P. Knauer, C. Sorce, V. Yu. Glebov, C. Stoeckl, T. W. Phillips, R. J. Leeper, K. Fletcher, and S. Padalino, Rev. Sci. Instrum. 74, 975 (2003).

[4] D. S. Hey, M. H. Key, A. J. Mackinnon, A. G. MacPhee, P. K. Patel, R. R. Freeman, L. D. VanWoerkom, and C. M. Castaneda, Rev. Sci. Instrum. 79, 053501 (2008).

[5] A. Mančić, J. Fuchs, P. Antici, S. A. Gaillard, and P. Audebert, Rev. Sci. Instrum. 79, 073301 (2008); H. Chen, N. L. Back, T. Bartal, F. N. Beg, D. C. Eder, A. J. Link, A. G. MacPhee, Y. Ping, P. M. Song, A. Throop, and L. Van Woerkom, ibid. 79, 033301 (2008).

[6] R. Prasad, D. Doria, S. Ter-Avetisyan, P. S. Foster, K. E. Quinn, L. Romagnani, C. M. Brenner, J. S. Green, P. Gallegos, M. J. V. Streeter, D. C. Carroll, O. Tresca, N. Dover, C. A. J. Palmer, J. Schreiber, D. Neely, Z. Najmudin, P. McKenna, M. Zepf and M. Borghesi, Nucl. Instr. Meth. Phys. Res. A 623, 712 (2010).

[7] R. Prasad, F. Abicht M. Borghesi, J. Braenzel, P. V. Nickles, G. Priebe, M. Schnurer and S. TerAvetisyan, Rev. Sci. Instrum. 84, 053302 (2013).

[8] SRIM - Stopping and Range of Ions in Matter, Ed. by J.F. Ziegler, J.P. Biersack, and M.D. Ziegler, (SRIM Co., Ion Implantation Press, 2008)

[9] T. J. Yu, S. K. Lee, J. H. Sung, J. W. Yoon, T. M. Jeong and J. Lee, Opt. Express 20, 10807 (2012).

[10] APD 1 PS 75/12/10/8 I 60:1 NR 8"FM P43, PHOTONIS USA, Inc., www.photonis.com

[11] D. Nikezic and K. N. Yu, Mater. Sci. Eng. R-Rep. 46, 51 (2004).

[12] A. Alejo, et al. Rev. Sci. Instrum. 85, 093303 (2014).

[13] R.J. Beuhler, L. Friedman, J. Appl. Phys. 48, 3928 (1977). 


\section{FIGURES}

Fig. 1. The schematic of the experimental arrangement. The unit of distance is $\mathrm{cm}$.

Fig. 2. The parabolic traces of ions, simultaneously recorded on the a) CR-39 and the b) MCP. Inset in a) shows the $\mathrm{C}^{6+}$ pits on CR-39. The zero point signal in b) indicated by the arrow is the emission from the source unaffected by the electric and magnetic fields of the spectrometer (neutral particles and $\mathrm{x}$-rays). These data are obtained for summed three consecutive shots.

Fig. 3. a) Parabolic ion traces recorded on the MCP behind slotted CR-39 plate and the proton pits on rear surface of first layer and front surface of second layer of CR-39 stack. b) Counted number of protons on the rear surface of first layer and front surface of second layer of CR-39 stack together with proton transmittance through $1 \mathrm{~mm}$ CR-39 plate.

Fig. 4. Absolute number of protons counted on the stripes of slotted CR-39 plate and the counts on CCD image of the MCP phosphor screen. These data are obtained for summed three consecutive shots.

Fig. 5. The parabolic traces of ions, simultaneously recorded on the a) CR-39 and the b) MCP are shown. Inset in b) shows the proton trace recorded by MCP. At energies $10 \mathrm{MeV}<E<12.6 \mathrm{MeV}$, and above $18 \mathrm{MeV}$ protons have passed through $1 \mathrm{~mm}$ CR-39 plate. The yellow arrow indicates proton signal on the MCP which has passed through the first CR39. The zero point signal indicated by the arrow is the emission from the source unaffected by the electric and magnetic fields of the spectrometer (neutral particles and x-rays). These data are obtained for a single shot.

Fig. 6. The calibration analysis results for protons, for both cases: single shot and three shot accumulation. The theoretical calculation fits well with the experimental data points.

Fig. 7. Calibration analysis results for carbon ions together with theoretical model calculations.

Fig. 8, a) CCD image of the MCP phosphor screen obtained from a single laser shot, and b) absolutely calibrated proton and $\mathrm{C}^{6+}$ spectra employing the measured calibration factors for protons (Fig. 6) and carbon ions (Fig. 7b)). 
(a)

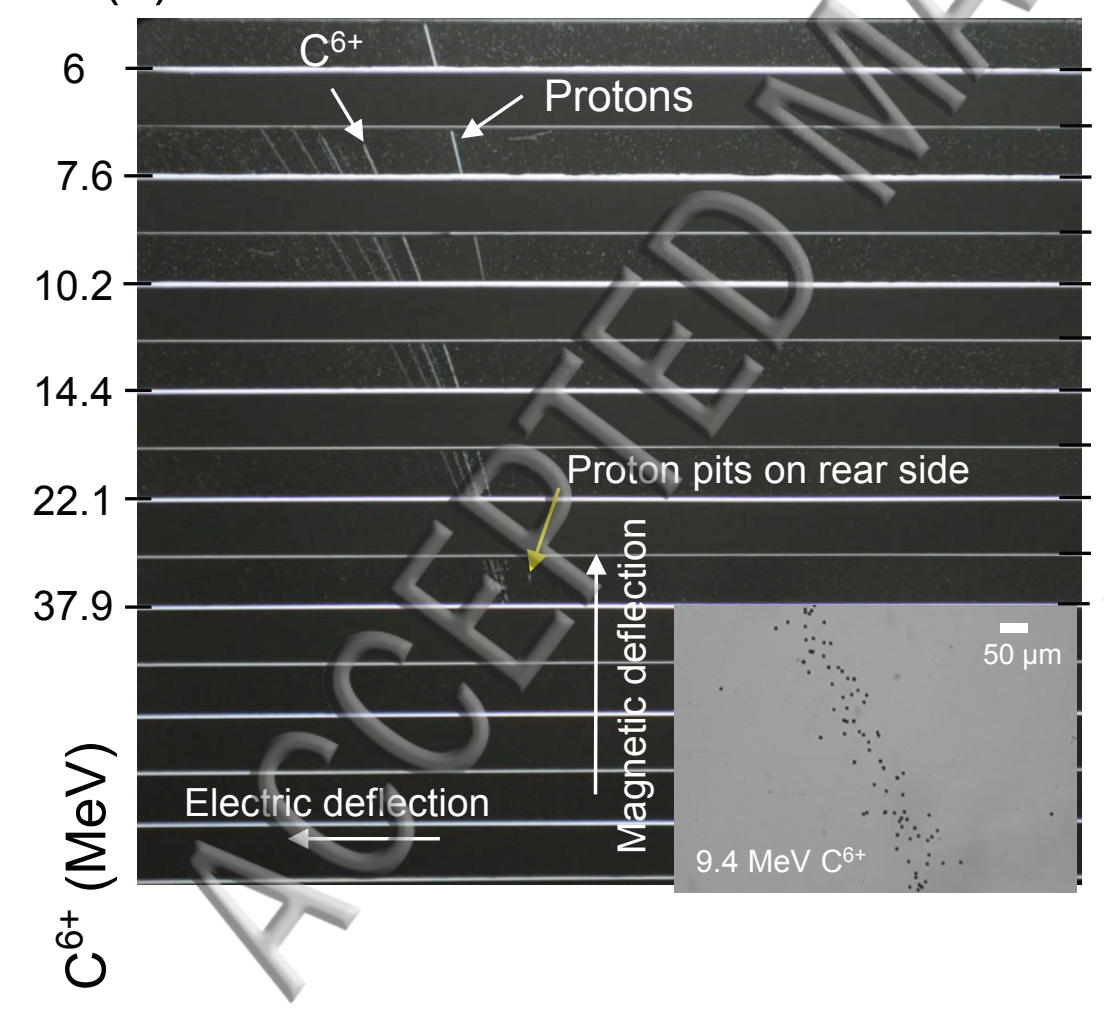

(b)

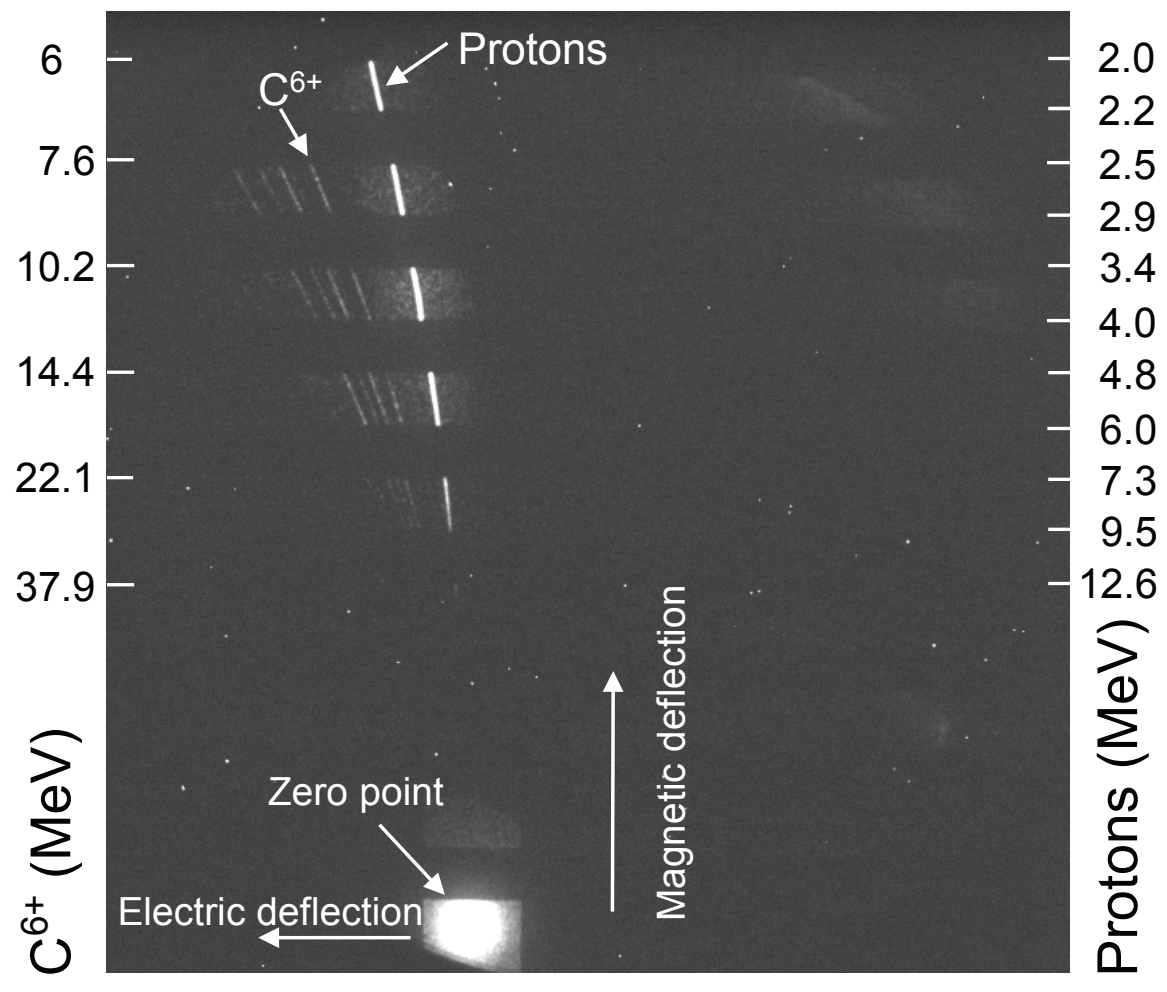

Fig. 2 
Fig. 3

(a)
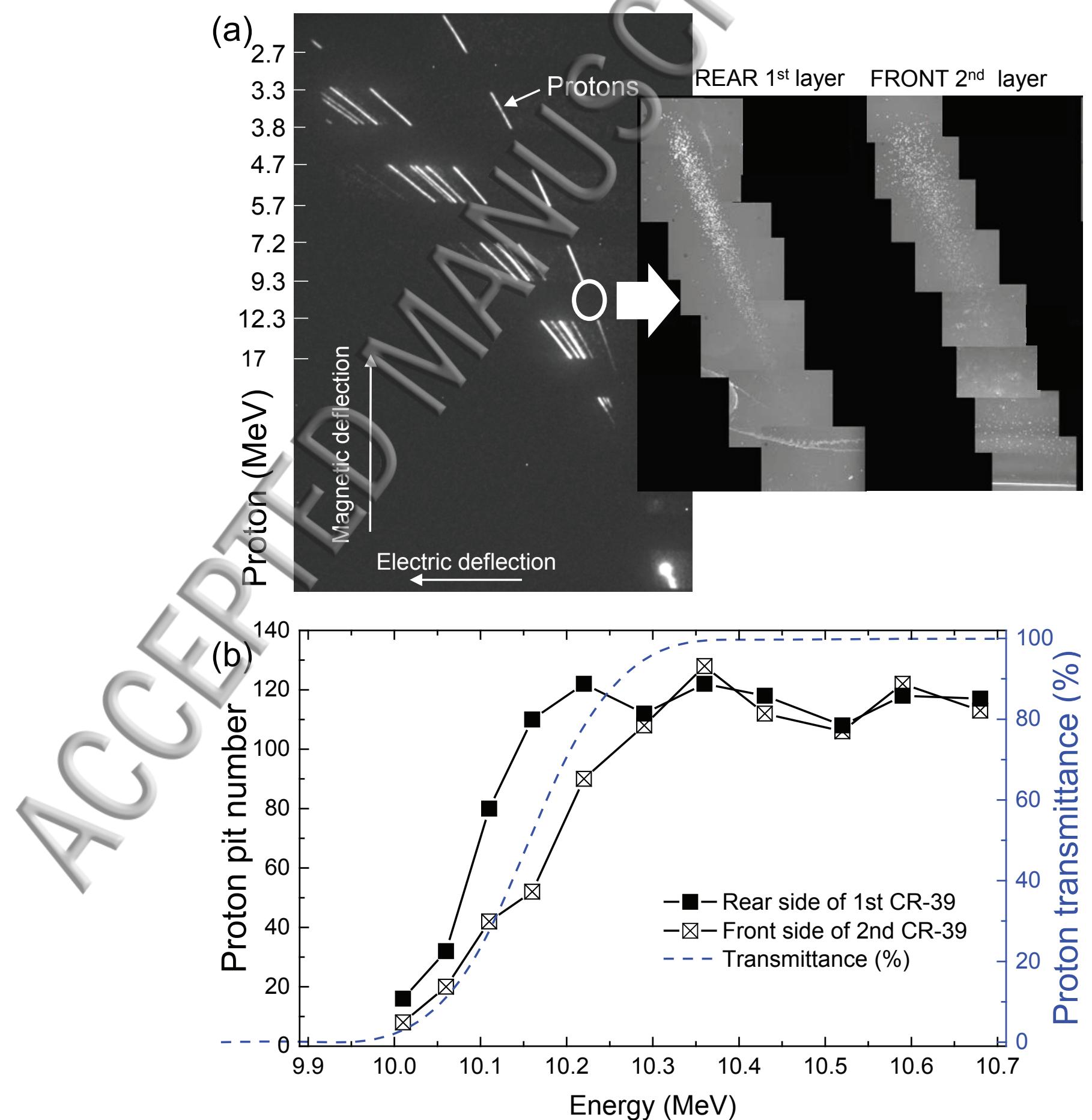


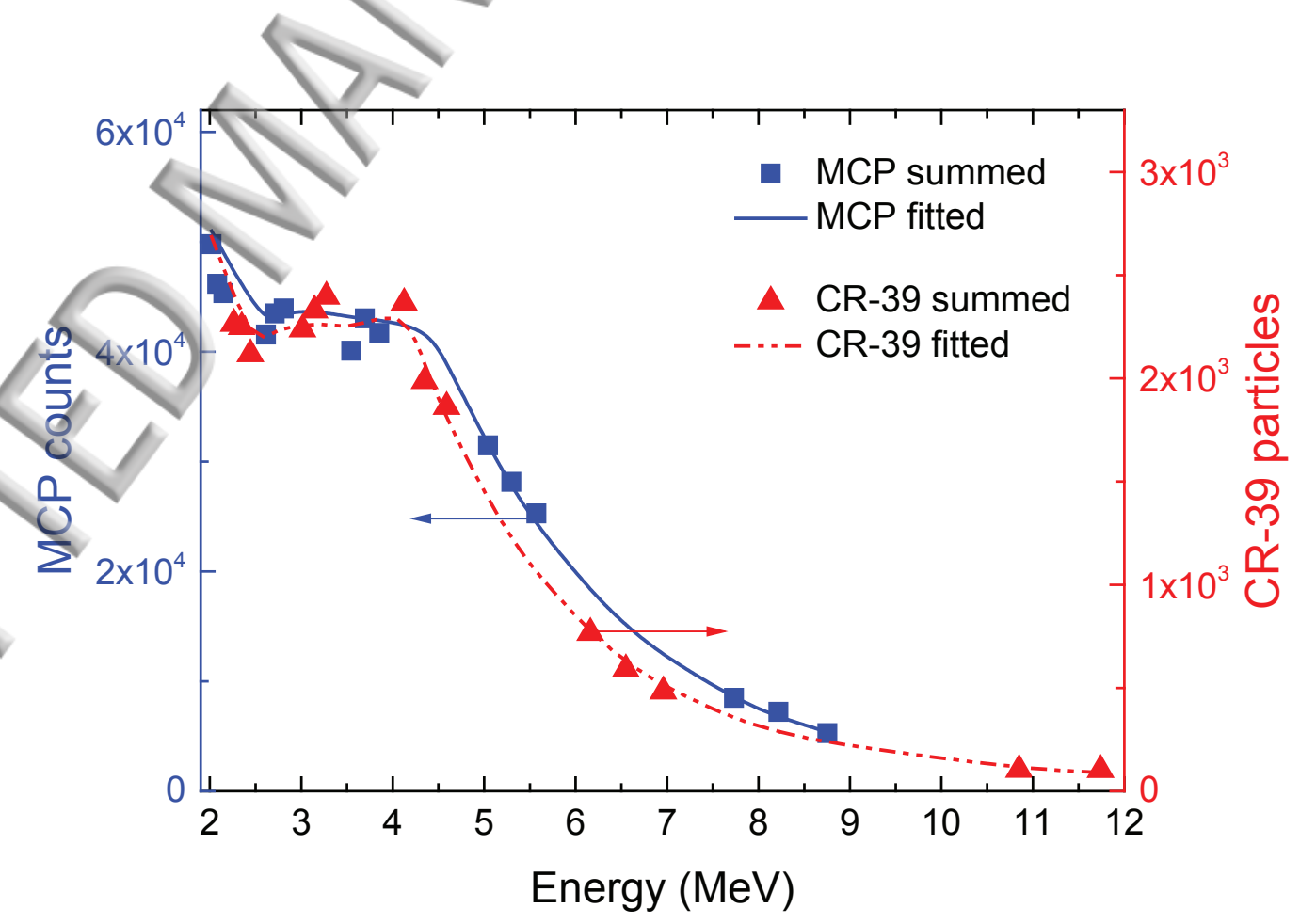

Fig. 4 


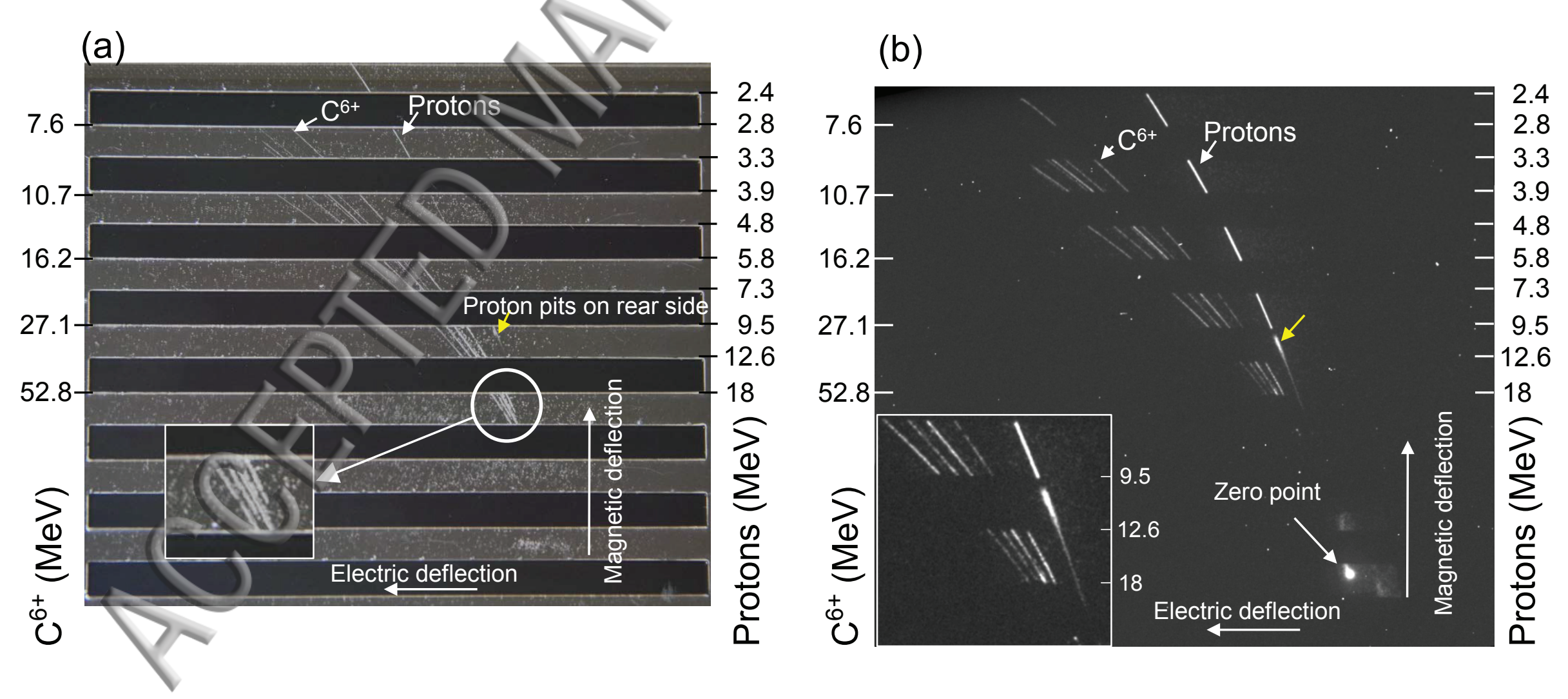

Fig. 5 


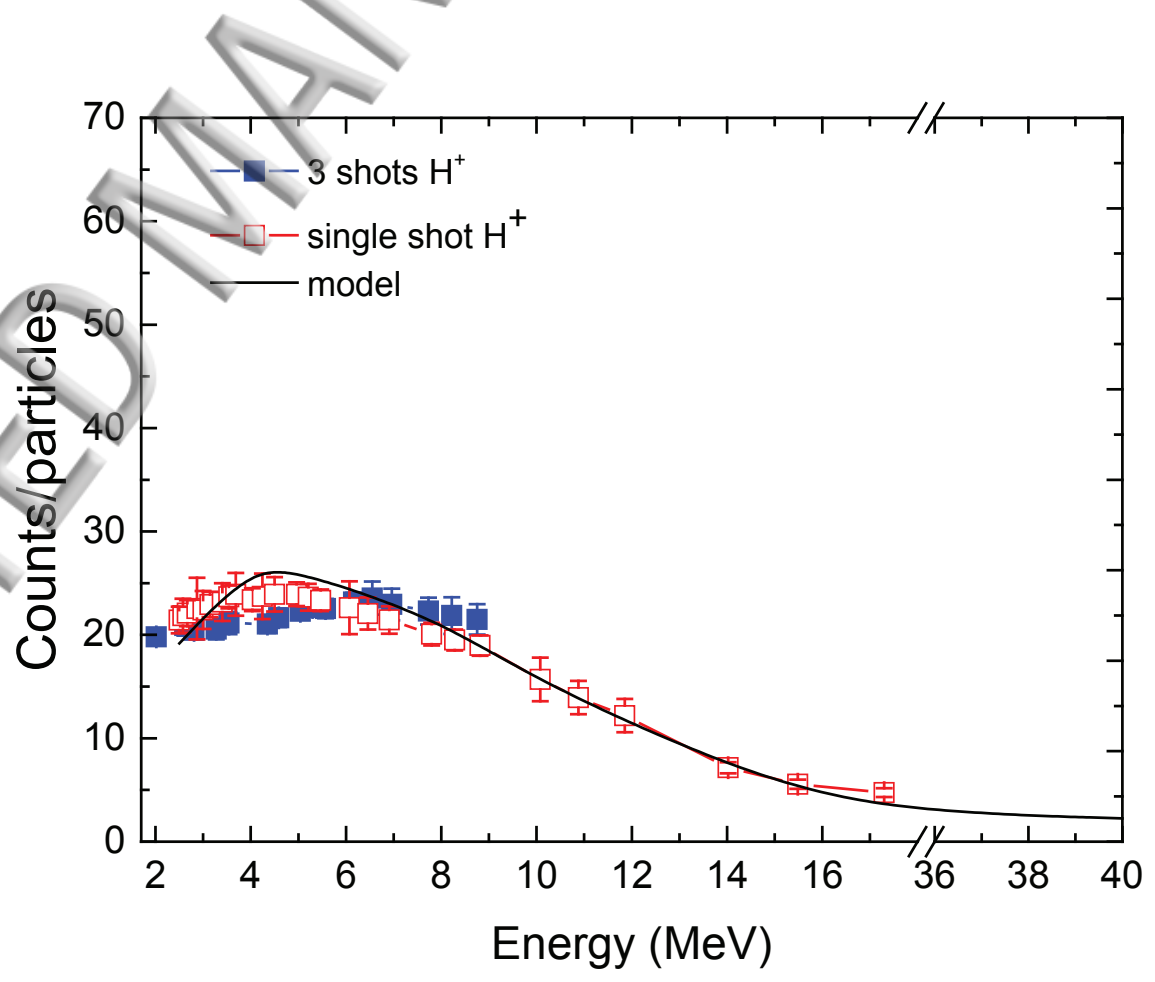

Fig. 6 


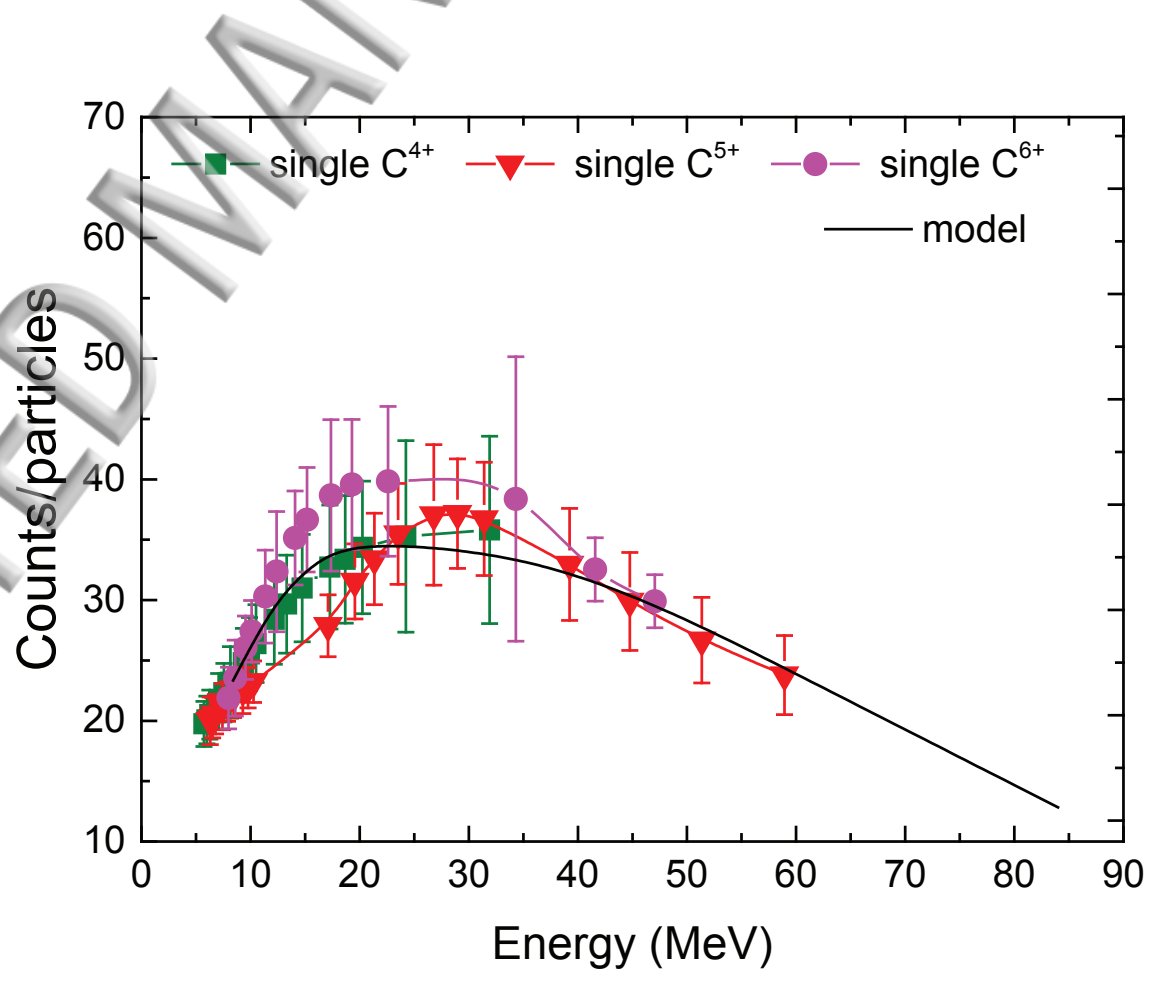

Fig. 7 
(a)
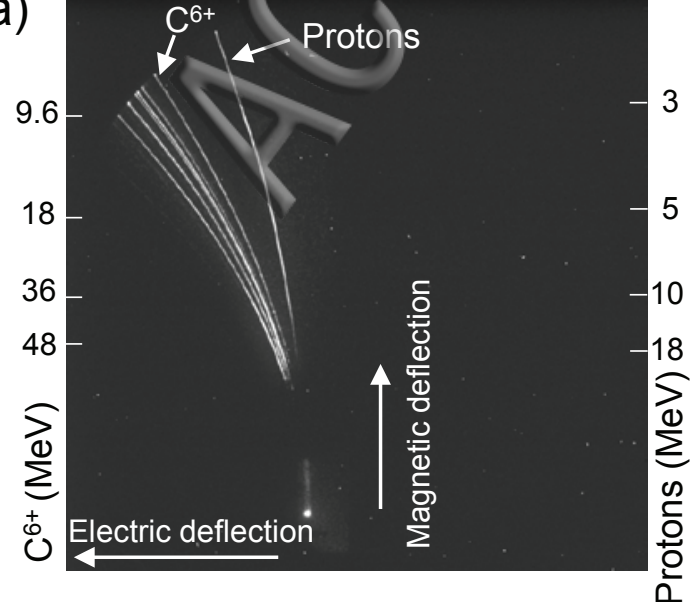

(b)

\section{(6)}

몸

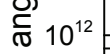

응

京

๕

흘

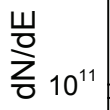

.

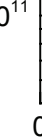

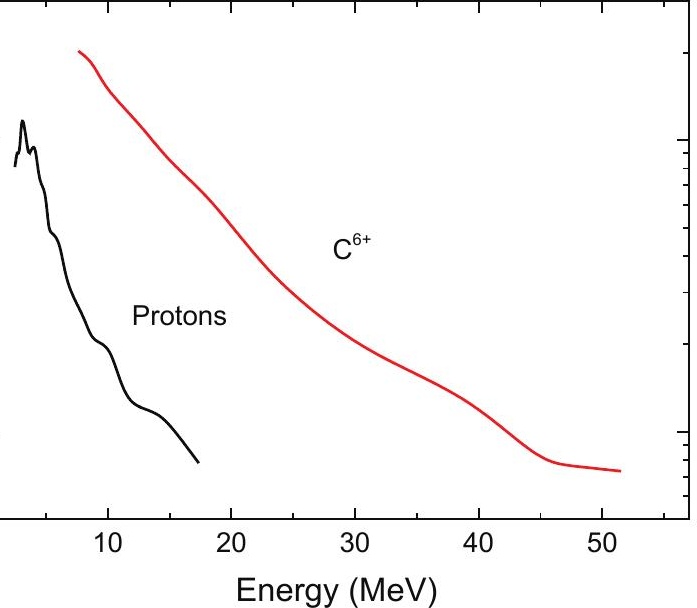

